\title{
Effect of Pharmacologically Induced Retinal Degeneration on Retinal Autofluorescence Lifetimes in Mice
}

\section{Authors: \\ Chantal Dysli (MD), Muriel Dysli (MD), Martin S. Zinkernagel (MD, PhD) and Volker Enzmann (PhD)}

Department of Ophthalmology and Department of Clinical Research, Inselspital, Bern University Hospital, University of Bern, Switzerland

\section{Corresponding Author:}

Volker Enzmann, PhD

Department of Ophthalmology

University of Bern

3010 Bern, Switzerland

volker.enzmann@insel.ch

Word count: 3800 (main script)

\section{Financial Support:}

This work was partly supported by a grant of the Swiss National Science Foundation (SNSF; MZ: \#320030_156019). The sponsor or funding organization had no role in the design or conduct of this research.

\section{Keywords:}

FLIO; fluorescence lifetimes; fundus autofluorescence; ophthalmic imaging; retinal degeneration; sodium iodate $\left(\mathrm{NalO}_{3}\right) ; \mathrm{N}$-methyl-N-nitrosourea (MNU) 


\section{Short title:}

Autofluorescence lifetimes in mice with pharmacological retinal degeneration

\section{Abbreviations:}

FAF: fundus autofluorescence; FLIO: fluorescence lifetime imaging ophthalmoscopy; OCT: optical coherence tomography; $\mathrm{NaIO}_{3}$ : sodium iodate, $\mathrm{MNU}$ : $\mathrm{N}$-methyl-N-nitrosourea; RPE: retinal pigment epithelium

\section{Abstract}

Fluorescence lifetime imaging ophthalmoscopy (FLIO) was used to investigate retinal autofluorescence lifetimes in mouse models of pharmacologically induced retinal degeneration over time. Sodium iodate $\left(\mathrm{NaIO}_{3}, 35 \mathrm{mg} / \mathrm{kg}\right.$ intravenously) was used to induce retinal pigment epithelium (RPE) degeneration with subsequent loss of photoreceptors (PR) whereas $\mathrm{N}$-methyl-N-nitrosourea (MNU, $45 \mathrm{mg} / \mathrm{kg}$ intraperitoneally) was employed for degeneration of the photoreceptor cell layer alone. All mice were measured at day $3,7,14$, and 28 after the respective injection of $\mathrm{NaIO}_{3}, \mathrm{MNU}$ or $\mathrm{NaCl}$ (control). Fluorescence lifetime imaging was performed using a fluorescence lifetime imaging ophthalmoscope (Heidelberg Engineering, Heidelberg, Germany). Fluorescence was excited at $473 \mathrm{~nm}$ and fluorescence lifetimes were measured in a short and a long spectral channel (498-560 nm and 560-720 nm). Corresponding optical coherence tomography (OCT) images were consecutively acquired and histology was performed at the end of the experiments. Segmentation of OCT images and histology verified the cell type-specific degeneration process over time. Retinal autofluorescence lifetimes increased from day 3 to day 28 in mice after $\mathrm{NalO}_{3}$ treatment. Finally, at day 28, fluorescence lifetimes were prolonged by $8 \%$ in the short and $61 \%$ in the long spectral channel compared to control animals ( $p=0.21$ and $p=0.004$, respectively). In mice after MNU treatment, the mean retinal autofluorescence lifetimes were already decreased at day 3 and retinal lifetimes were finally shortened by $27 \%$ in the short and $51 \%$ in the long spectral channel at day $28(\mathrm{p}=0.0028)$. In conclusion, degeneration of the RPE with subsequent photoreceptor degeneration by $\mathrm{NaIO}_{3}$ lead to longer mean fluorescence lifetimes of the retina compared to control mice, whereas during specific degeneration of the photoreceptor layer induced by MNU shorter lifetimes were measured. Therefore, short retinal fluorescence lifetimes may originate from the RPE and may be modified by the overlaying retinal layers. 


\section{Introduction}

Fluorescence lifetime imaging in ophthalmology (FLIO) recently emerged as an opportunity for detailed analysis of structural as well as metabolic changes within the retina. In principle, natural retinal fluorophores are excited using blue laser light and their electrons reach a higher energy level. This energy is then partly released in form of photons with longer wavelength and detected by retinal autofluorescence imaging. The measured autofluorescence can be quantified by its intensity as well as by the fluorescence lifetimes. Fluorescence lifetimes represent the time a molecule spends in its excited state before returning to its ground state. These lifetimes are specific for each fluorophore and can be modified by the fluorophore's microenvironment namely the temperature, $\mathrm{pH}$ and oxygenation status (Schweitzer et al. 2007). Therefore, in vivo measurement of fluorescence lifetime within the retina might be used for investigation of metabolic changes before irreversible structural changes occur. In previous reports, fluorescence lifetimes have been characterized in mice (Dysli et al. 2014a) and the healthy human retina (Dysli et al. 2014b) as well as in specific retinal diseases like retinal artery occlusion, Stargardt disease and age related macular degeneration (Dysli et al. 2014b, Dysli, Wolf and Zinkernagel 2015b, Dysli et al. 2016a, Dysli, Wolf and Zinkernagel 2016b). The main origin of fundus autofluorescence is the retinal pigment epithelium (RPE), containing a complex mixture of bisretinoid fluorophores summarized as lipofuscin (Sparrow et al. 2012);(Delori et al. 1995). Recently, the short autofluorescence lifetimes within the macular center were attributed to xanthophyll, showing a good correlation to the distribution of macular pigment within the fovea (Sauer et al. 2015). However, in vivo FLIO measurements from the fundus are assumed to comprise a sum of signals from different retinal layers as well as partly also from the crystalline lens. Differentiation of the contribution of individual layers to the measured mean fluorescence lifetime is not possible in the presence of intact retina. Therefore, a method to selectively address specific retinal layers is needed.

Previously, we established the FLIO technique for measurements in mouse models and characterized fluorescence lifetimes in different mouse strains (Dysli et al. 2014a). In the current study we aim to analyze the contribution of individual outer retinal layers to the measured fluorescence lifetime signal in vivo using two different models of pharmacologically induced retinal degeneration in C57BI/6 mice: sodium iodate $\left(\mathrm{NaIO}_{3}\right)$ and N-methyl-N-nitrosourea (MNU). Thereby, time dependent changes of retina autofluorescence lifetimes are investigated. 
$\mathrm{NalO}_{3}$ induces rapid degeneration of the RPE and is used as a model for RPE atrophy as seen in age-related macular degeneration (AMD) (Franco et al. 2009, Enzmann et al. 2006). There are different interaction modes described in literature for the toxic effect of $\mathrm{NalO}_{3}$ on the RPE: basal plasma membrane destruction (Sen et al. 1992), cross-reaction with melanin, leading to cytotoxic metabolites (Baich and Ziegler 1992), inhibition of crucial enzymes (Korte, Rappa and Andracchi 1991), and altered adhesion between the neurosensory retina and the RPE layer (Yoon and Marmor 1993). Subsequently, time- and concentration-dependent, the RPE atrophy and dysfunction leads to degeneration of the overlaying photoreceptor (PR) layer resulting in a decrease of the outer nuclear layer (ONL) (Yang et al. 2014). Additionally, direct toxic effects on the PR cells are also described recently (Wang et al. 2014).

In a second model, MNU was used, which specifically damages PRs in a concentration dependent manner (Tsubura et al. 2010). MNU acts through DNA adduct formation (Yoshizawa et al. 1999) and/or endoplasmatic reticulum stress (Reisenhofer et al. 2015). This leads to apoptosis within the PR resulting in a decrease of the thickness of the ONL. Therefore, MNU can serve as a model displaying general features of retinitis pigmentosa.

\section{Material and Methods}

\subsection{Mice}

Wild type C57BL/6 mice at the age of 6-8 weeks were obtained from the breeding facility of the Department of Clinical Research, University of Bern, Bern, Switzerland. During experimentation, all mice were housed under a standard 12-h:12-h light-dark cycle with food and water available ad libitum. All procedures were performed after governmental approval according to the Swiss Federal Regulations on Animal Welfare and were in accordance with the National Institutes of Health guide for the care and use of laboratory animals. All experiments are registered under the animal experimentation number BE127/12.

\subsection{Study Protocol}

26 mice were investigated with six animals in the control group and ten animals in each degeneration group. Data was acquired in two independent experimental series. Both eyes of all animals were investigated at day 3, 7, 14 and 28 after injection by the same experienced investigators. Every examination included sequential acquisition of fluorescence lifetime images (FLIO) and optical coherence tomography (OCT) scans. 


\subsection{Pharmacological Induction of Retinal Degeneration}

Retinal degeneration was induced according to previously published protocols (Franco et al. 2009). Sodium iodate $\left(\mathrm{NaIO}_{3}\right.$; Sigma-Aldrich, Switzerland) was dissolved in sterile $0.9 \%$ $\mathrm{NaCl}$ (B. Braun Medical AG, Sempach, Switzerland) and injected intravenously at a concentration of $35 \mathrm{mg} / \mathrm{kg}$ bodyweight (BW). N-methyl-N-nitrosourea (MNU; SigmaAldrich, Buchs, Switzerland) was dissolved in sterile $0.9 \% \mathrm{NaCl}$ containing $0.05 \%$ acetic acid (Fresenius Kabi, Oberdorf, Switzerland) and injected intraperitoneally at a concentration of $45 \mathrm{mg} / \mathrm{kg} \mathrm{BW}$. In the control group, animals were injected with $100 \mu \mathrm{l}$ $\mathrm{NaCl}$ intravenously or intraperitoneally, respectively.

\subsection{Animal Preparation for Imaging Procedures}

For image acquisition, all mice were anaesthetized by subcutaneous (s.c.) injection of 0.75 $\mathrm{mg} / \mathrm{kg}$ medetomidine (Domitor, $1 \mathrm{mg} / \mathrm{mL}$; Orion Pharma, Provet, Zug, Switzerland) and 45 $\mathrm{mg} / \mathrm{kg}$ ketamine (Ketalar $50 \mathrm{mg} / \mathrm{mL}$; Parke-Davis, Pfizer, Zürich, Switzerland). Not before 30 minutes after injection, anesthesia was reversed with $0.75 \mathrm{mg} / \mathrm{kg}$ atipamezole s.c. (Antisedan $5 \mathrm{mg} / \mathrm{mL}$; Pfizer). Additionally, $400 \mu \mathrm{L}$ of $0.9 \% \mathrm{NaCl}$ was injected s.c. to prevent dehydration. Pupils were maximally dilated using tropicamide $0.5 \%$ and phenylephrine $\mathrm{HCl} 2.5 \%$ eye drops (ISPI, Bern, Switzerland). To prevent corneal desiccation during image acquisition, methylcellulose (Methocel 2\%; OmniVision, Neuhausen, Switzerland) diluted 1:1 with balanced salt solution (Alcon, Schaffhausen, Switzerland) was applied onto the cornea.

\subsection{Fluorescence Lifetime Imaging Ophthalmoscope}

Fluorescence lifetimes were measured using a fluorescence lifetime imaging ophthalmoscope, based on a Heidelberg Engineering Spectralis system (Heidelberg Engineering, Heidelberg, Germany). The technique of fluorescence lifetime imaging ophthalmoscopy (FLIO) has been previously described in detail (Dysli et al. 2014b, Dysli et al. 2014a). The following section will provide a short summary of the technical background. A 25-diopter lens ( $f=40 /+25$ diopters; Heidelberg Engineering) was added in front of the FLIO device in order to adapt to the short axial length of the murine eye $(3.37 \mathrm{~mm}$, (Remtulla and Hallett 1985)). A confocal system with a $473 \mathrm{~nm}$ pulsed laser was used for exciting the endogenous retinal autofluorescence of the central retina. The emitted fluorescence photons were detected by two highly sensitive hybrid photon-counting detectors (HPM-100-40, Becker \& Hickl, Berlin, Germany) in a short (498 to $560 \mathrm{~nm}$ ) and 
in a long (560 to $720 \mathrm{~nm}$ ) spectral channel and registered by time-correlated single-photon counting (TCSPC) modules (SPC-150; Becker \& Hickl). Data was individually recorded for every single pixel within a 256x256 pixel grid. An infrared reflectance image was used for tracking of eye movements. This ensured that acquired fluorescence intensity and lifetime data were recorded at the correct spatial location and allowed for data accumulation over the scan duration of about 2-3 minutes that was required for the FLIO measurement (minimum of 700-1000 photons per pixel). Thereby, an effect of photo pigment bleaching can be excluded because it occurs within the first 20 seconds and FLIO results are averaged over a much longer acquisition period (Delori et al. 2011). The recorded lifetime data was bi-exponentially fitted by Becker \& Hickl software (SPCImage 4.6) using a binning factor of one. An incomplete multiexponential decay model was applied and tail fit was started at the peak of fluorescence. In both, the short and the long spectral channel, each pixel is characterized by a short and a long lifetime component $T 1$ and $T 2$ and their respective amplitudes $\alpha 1$ and $\alpha 2$. Tm (Equation 1) reflects the amplitude-weighted mean fluorescence lifetime value and was used for further analysis.

Equation 1: $\quad T m=\frac{\alpha 1 * T 1+\alpha 2 * T 2}{\alpha 1+\alpha 2}$

\subsection{Optical Coherence Tomography Image Acquisition}

Optical coherence tomography (OCT) images were acquired using the Heidelberg Retina Angiograph [HRA] Spectralis system (Heidelberg Engineering, Heidelberg, Germany). OCT imaging was performed according to previous protocols (Dysli et al. 2014a, Ebneter et al. 2015, Dysli et al. 2015a). The murine eye was covered with a contact lens ( $9 \mathrm{~mm}$ diameter; base curve 7.2; power ( $\left.F^{\prime}\right)+4$ diopters; Bausch \& Lomb; OmniVision) and a 78 diopter lens (Volk Optical, Inc., Mentor, OH, USA) was added in front of the OCT camera. At every examination, an OCT volume scan (automatic real-time mode) centered on the optic nerve head was acquired from every eye.

\subsection{OCT Analysis}

Individual retinal layers within the OCT volume scan were automatically segmented by the inbuilt segmentation algorithm from the Heidelberg Eye Explorer (Version 1.9.10; Heidelberg Engineering, Germany). Automatic segmentation was manually verified and corrected where needed. The total retinal thickness was measured from the inner limiting 
membrane (ILM) until the basement membrane (BM). Additionally, the boundary between the outer plexiform layer (OPL) and outer nuclear layer (ONL) was segmented. It builds the border between the outer retina with the PR cell layer and the inner retinal layers. Corresponding thickness volume maps were automatically generated by the Heidelberg Eye Explorer.

\subsection{Histology}

At day 28 , histology was performed with all retinas. For this purpose, eyes were fixed in $4 \%$ paraformaldehyde in phosphate buffered saline at $4^{\circ} \mathrm{C}$ overnight. Afterwards, samples were gradually dehydrated in an alcohol series, embedded in paraffin, cut in $5 \mu \mathrm{m}$ sections through the optic nerve head and mount on glass slides. Histological sections were stained with hematoxylin and eosin stain (H\&E).

In order to visualize lipofuscin accumulation during induced retinal degeneration, paraffin cross sections of control, $\mathrm{MNU}$ treated and $\mathrm{NaIO}_{3}$ treated mice were stained with Nile Blue sulfate. Thereby, the slides were dyed with saturated Nile Blue A solution (Sigma-Aldrich; $1 \mathrm{mg} / \mathrm{ml}$ ) for 30 minutes followed by $48 \mathrm{~h}$ bleaching with $10 \% \mathrm{H}_{2} \mathrm{O}_{2}$ (Sigma-Aldrich) to discriminate between lipofuscin-like material and melanin (Hueck 1912, Lillie 1956).

\subsection{Statistical Analysis}

Fluorescence lifetime data and retinal thickness data were analyzed within the areas of the standard Early Treatment Diabetic Retinopathy Study (ETDRS) grid which was manually centered on the optic nerve head (Dysli et al. 2014a). For FLIO data, the custom made "FLIO reader" (ARTORG Center for Biomedical Engineering Research, University of Bern, Switzerland) was used. OCT data was taken over from the thickness map analysis within the Heidelberg explorer. Mean values of the inner ring (corresponding to approximated diameter of 600-1800 $\mu \mathrm{m}$ ) were further analyzed. Note that for OCT analysis measurements of lateral distances might not be accurate since the dimensions of the mouse eye differ significantly from a human eye and additional lenses were used. However, axial OCT measurements seem to be accurate (Lozano and Twa 2013).

For statistical analysis, corresponding data from the right and the left eye were averaged for every mouse, resulting in one single mean value per area and modality for each animal.

Statistical analysis was performed using Prism software version 6 (GraphPad Software, Inc., La Jolla, CA, USA). Mean values are compared by Mann Whitney test (two-tailed, confidence interval $95 \%$, significance level $=0.05$ ) and coefficient of variation $(C V)$ within 
the groups was assessed. Differences between the groups were calculated using KruskalWallis test for non-parametric data and Dunn's multiple comparison test for post-test analysis. Results are presented as mean \pm standard error of the mean (SEM).

\section{Results}

\subsection{Retinal autofluorescence lifetimes in $\mathrm{MNU}$ and $\mathrm{NalO}_{3}$ degeneration models}

Examples of fundus autofluorescence lifetime maps at day 28 are shown in Figure 1 in order to visualize the different lifetime values in the investigated models. Mean retinal autofluorescence lifetime values in control animals were $1171 \pm 14 \mathrm{ps}$ in the short spectral channel (SSC) and $385 \pm 16$ ps in the long spectral channel (LSC; Figure 2). Differences between the three groups were more pronounced in the LSC. In mice treated with MNU, the mean fluorescence lifetime was significantly shortened to $924 \pm 24$ ps in the SSC and $294 \pm 8 p s$ in the LSC at day 28 ( $p=0.0028$ and 0.0069 , respectively). A similar tendency was already measured at day three after injection. In mice treated with $\mathrm{NalO}_{3}$, the mean fluorescence lifetime was prolonged in both spectral channels to $1266 \pm 56$ ps in the SSC and $621 \pm 38 p s$ in the LSC at day 28 ( $p=0.2$ and 0.0085 , respectively). In the LSC the mean lifetime value slightly increased over time between the measurements at day three and day 28. The lifetime values within the $\mathrm{NalO}_{3}$ group displayed a higher variability compared to the control and the MNU group [CV: 13\% (SSC) resp. 17\% (LSC) compared to $8 \%$ resp. $8 \%$ in the $\mathrm{MNU}$ and $2 \%$ resp. $8 \%$ in the control group].

\subsection{Analysis of individual lifetime components}

The investigated mean fluorescence lifetime value is composed of the short lifetime component $T 1$ and the long component $T 2$ as described above (Equation 1). In the long spectral channel, where the differences between the experimental groups were the biggest, further analysis of the distribution histogram (T1 versus T2) revealed a shift of both components towards shorter lifetimes in the $\mathrm{MNU}$ model (Figure 3). In $\mathrm{NalO}_{3}$ treated mice, $T 1$ values were clearly prolonged whereas $T 2$ values were similar as in the control group.

\subsection{OCT analysis of retinal degeneration}

OCT images of the retinal structures in control and treatment groups revealed toxin-related changes in the retinal architecture. Representative examples are shown in Figure 4. Total retinal thickness significantly decreased in both degeneration models from $227 \pm 2 \mu \mathrm{m}$ (control group) to $124 \pm 2 \mu \mathrm{m}$ in the $\mathrm{MNU}$ group and $137 \pm 6 \mu \mathrm{m}$ in the $\mathrm{NaIO}_{3}$ group at day 
28 (Figure 5). In MNU treated mice, retinal degeneration occurred for the most part in the first seven days after injection (decrease by $39 \%$ compared to control animals) with a decrease of $50 \mu \mathrm{m}(22 \%)$ already at day three, $43 \%$ at day 14 , and a final decrease of $46 \%$ at day $28 . \mathrm{NaIO}_{3}$ treated mice showed a slower decrease in retinal thickness with $1 \%$ decrease at day three, $22 \%$ at day seven, $32 \%$ at day 14 and finally $40 \%$ decrease at day 28 compared to the controls. Additionally, the measured range of retinal thickness values was broader scattered in $\mathrm{NalO}_{3}$ treated mice than in MNU treated and control mice.

In order to link the observed changes in fluorescence lifetimes to the induced degeneration, we segmented the OCT images to analyze the influence of degeneration of specific retinal layers on the retinal autofluorescence lifetimes. Both degeneration models showed a degeneration of the PR cell layer. Therefore, the total retinal thickness highly correlated with the PR thickness $\left(\mathrm{NaIO}_{3}: \mathrm{R}^{2}=0.98\right.$ and $\mathrm{MNU}: \mathrm{R}^{2}=0.998$; Figure 5$)$. In the $\mathrm{NaIO}_{3}$ model a slower decrease in the PR thickness was measured when compared to the MNU model. At day 28, in both models, a slight decrease in the thickness of the inner retinal layers was observed (both models: $-21 \mu \mathrm{m}$ or $19 \%$ ). In $\mathrm{NalO}_{3}$ treated mice, the degeneration of the RPE was confirmed by OCT. At day seven and 14, a decreasing number of remaining patches of RPE were still visible (Figure 6, blue arrows). At day 28, there was no hyperreflective RPE band above the choroid identifiable. In the MNU model, the hyperreflective RPE was clearly distinguishable from the choroid at all measure points. However, in this model, the rapid PR degeneration led to a separation of the inner retinal layers from the RPE and a subretinal fluid accumulation (Figure 6, red asterisks).

\subsection{Histological analysis}

Histological sections of the murine retina at day 28 confirmed the cell type-specific retinal degeneration and corresponded to the respective OCT scans (Figure 4). In the unaffected control mice, expectedly the retinal layers remained normal. In MNU treated mice, the OPL was absent, and the ONL and the ellipsoid zone (IS/OS) was no longer identifiable. However, in contrast to the $\mathrm{NalO}_{3}$ treated mice, the RPE/BM was well preserved. In the $\mathrm{NalO}_{3}$ model, the RPE/BM was discontinuous with patchy RPE loss and irregular remaining cell clots. The ellipsoid zone was largely not detectable. The ONL was clearly rarefied and its thickness was substantially reduced compared to the control animals.

In order to substantiate if an accumulation of lipofuscin could explain the changes in lifetime imaging values we have performed Nile Blue A staining at day 28 . Thereby, positive blue staining in the area of retinal damage in $\mathrm{NalO}_{3}$ treated mice indicates an accumulation of the compound (see Figure 7). 


\section{Discussion}

Fluorescence lifetime imaging ophthalmoscopy is used for investigation of structural and metabolic changes within the retina. In animal models of pharmacologically induced retinal degeneration, significant changes of mean retinal fluorescence lifetime values were observed. MNU lead to degeneration of the PR and to significantly shortened fluorescence lifetimes whereas $\mathrm{NaIO}_{3}$ induced RPE and subsequent PR degeneration which caused prolonged lifetimes.

Retinal autofluorescence mainly originates within the RPE and measurement of the fundus autofluorescence intensity upon excitation with specific laser light is widely used for diagnostic purposes as well as for follow up examinations in various retinal diseases. In autofluorescence intensity measurements, lipofuscin and its derivates dominate possible other weak fluorophores. However, as fluorescence lifetimes in general are independent of the fluorophores concentration, also fluorophores with weak autofluorescence intensity properties can influence the fluorescence lifetime measurements (Schweitzer 2010, Becker 2012).

Previous FLIO measurements in acute retinal artery occlusion with reduced oxygen supply and massive swelling of the inner retinal layers revealed prolonged fluorescence lifetimes (Dysli et al. 2015b). However, in the post-acute phase with atrophy of the inner retinal layers, no change in fluorescence lifetime measurement was detected compared to the healthy control eyes. Therefore, we assume that the inner retinal layers in normal oxidative conditions do not influence FLIO. The contribution of the outer retinal structures, namely the PR and the RPE, has not been investigated yet. To increase the knowledge in this matter we have employed two mouse models with distinct pharmacologically induced changes in these tissues.

Our current study results suggest two hypotheses explaining the observed changes in fluorescence lifetimes during retinal degeneration. Isolated PR degeneration leads to shortened lifetimes. Thereby, the normal, now absent PR cell layer itself might either exhibit long lifetimes or attenuate the contribution of short signals from the underlying RPE. Therefore, the measured shorted lifetimes could be attributed to the intact RPE. In the model with primary RPE degeneration, missing contribution of these short RPE lifetimes could lead to a general prolongation of the mean retinal fluorescence lifetimes. This might result from a proportional increase of contribution of longer lifetimes from other retinal layers and possibly also from the underlying choroid. 
Another explanation could be that the RPE itself changes its fluorescence lifetime properties during the MNU-induced degeneration process. Isolated destruction of the PR induces large amounts of cell debris and activates the RPE as seen in Stargardt disease (Dysli et al. 2016a). Therewith the environment of the RPE is changed and, correspondingly, FLIO values originating in the cells are altered.

Alternatively, metabolites themselves arising from the PR degeneration process could temporarily contribute to short mean fluorescence lifetimes (e.g., lipofuscin; Figure 7). However, the conclusion of the Nile Blue A staining has to cautioned because this method on its own is not sufficient to detect lipofuscin unequivocally. Additional methods (e.g., autofluorescence, HPLC) might strengthen the evidence. Over time and with progressing degeneration this effect is thought to be decreasing. Such a development was not observed within the experimental period of 28 days. Short time accumulation of metabolic by- or end-products within the visual cycle, namely all-trans retinal, A2E and other fluorophores of lipofuscin with fluorescence lifetimes of 31 ps (Loguinova et al. 2009), 189 ps (Schweitzer 2010) and 385 ps (Schweitzer et al. 2012) could lead a relative increase of short lifetime components. As the general mean lifetime value in the murine fundus is basically significantly longer compared to the human retina (SSC: $1171 \pm 14$ ps vs. $255 \pm 6$ ps; LSC: $385 \pm 16$ ps vs. $282 \pm 4$ ps) (Dysli et al. 2014a), an increase of components with short lifetimes would have a larger effect. On the mitochondrial level, the redox pair FAD/FADH2 (oxidized and reduced flavin adenine dinucleotide), which theoretically exhibits excitation and emission spectra within the range of the FLIO system, might be shifted toward the oxidized FAD (Islam et al. 2013, Schweitzer et al. 2007). Oxidized flavin adenine dinucleotide is shown to have very short fluorescence lifetimes within the range of $2.5 \mathrm{~ns}$ in its free form and 150 ps in the bound form (Skala et al. 2007). However, if the RPE and its metabolic activity are lost as seen in the $\mathrm{NalO}_{3}$ model, the short lifetime components will disappear.

Interestingly, changes in retinal fluorescence lifetimes were already detected at day three in both degeneration models. At this point in time OCT measurements in the $\mathrm{NaIO}_{3}$ degeneration model revealed only slight reduction of the total retinal thickness and no major changes in the retinal architecture (see also Figure 6c). This leads to the assumption that retinotoxic effects can be detected by FLIO even before structural changes seen by OCT occur. Therefore, FLIO could be an appropriate tool to detect early metabolic changes within the retina and potentially provide early diagnostic values. 
However, our study comprises some limitations. The conditions within the FLIO device are predefined and no adaptation of excitation wavelength or adjustment of the detection wavelengths range is possible. Both changes would be of interest to focus on specific fluorophores. Furthermore, FLIO is not conceived for quantitative autofluorescence and therefore, direct quantification of autofluorescence intensity with FLIO is not possible. Additionally, this study was performed at time points and with dosages of $\mathrm{NaIO}_{3}$ and $\mathrm{MNU}$ which are known to clearly induce toxic effects on the retina. Reduced dosages and earlier time points might provide more detailed information about the metabolic changes induced by those two compounds. Furthermore, reactive Müller cells and invading microglia/macrophages could also influence the recorded fluorescence lifetimes. We could show earlier that in both models of retinal degeneration used in this study the Müller cells are activated. Others have published similar observations (Wan et al. 2008, Kiuchi et al. 2002). In regard to microglia/macrophages an increase of such cells after treatment with MNU or $\mathrm{NaIO}_{3}$ respectively, has been described (Kaneko et al. 2008, Machalinska et al. 2014). Because the measurements were performed in the eye as a whole system, fluorescence lifetime measurements were mainly of descriptive origin and direct conclusions to single fluorophores are not possible by now. Therefore, further investigations are much needed for the identification of individual molecular and metabolic contributors to the measured mean fluorescence lifetime data.

In summary, the two investigated degeneration models reveal interesting characteristics about the contribution of individual retinal layers to the measured sum of fluorescence lifetime signals due to changes in their metabolism.

\section{Conclusion}

By using pharmacologically induced ablation of specific retinal layers, the origin of autofluorescence lifetimes of the retina can be dissected. Ultimately this will help to understand autofluorescence lifetime patterns seen in patients with retinal degeneration. Degeneration of the RPE with subsequent photoreceptor degeneration by $\mathrm{NaIO}_{3}$ lead to longer mean fluorescence lifetimes of the retina compared to control mice, whereas in solely degeneration of the photoreceptor layer induced by MNU shorter lifetimes are measured. Therefore, short retinal fluorescence lifetimes may originate from the RPE and may be modified by the adjacent retinal layers. 


\section{Acknowledgement}

This work was partly supported by a grant of the Swiss National Science Foundation (SNSF; MZ: \#320030_156019). The fluorescence lifetime imaging ophthalmoscope is kindly provided and supported by Heidelberg Engineering (Heidelberg, Germany). The sponsor or funding organization had no role in the design or conduct of this research. The authors also thank Monika Kilchenmann for technical assistance. 
Table 1: Abbreviations

Index of abbreviations of retinal layers used in text and figures.

\begin{tabular}{|c|c|}
\hline \multicolumn{2}{|c|}{ Abbreviations } \\
\hline BM & basement membrane \\
\hline ELM & external limiting membrane \\
\hline GCL & ganglion cell layer \\
\hline INL & inner nuclear layer \\
\hline IPL & inner plexiform layer \\
\hline IS/OS & inner/outer segment (ellipsoid zone) \\
\hline ONL & outer nuclear layer \\
\hline OPL & outer plexiform layer \\
\hline RNFL & retinal nerve fiber layer \\
\hline RPE & retinal pigment epithelium \\
\hline
\end{tabular}


Figure legends:

Figure 1: Retinal autofluorescence lifetime map of the murine eye (A, control) and after pharmacologically induced retinal degeneration with $\mathrm{MNU}(\mathrm{B})$ and $\mathrm{NaIO}_{3}(\mathrm{C})$. Fundus autofluorescence intensity and fluorescence lifetime images at day 28 after injection in the short and long spectral channel $(S S C=498-560 \mathrm{~nm}, \mathrm{LSC}=560-720 \mathrm{~nm}$ ) with corresponding fluorescence lifetime distribution histograms of these individual FLIO maps (D). (Color range SSC: 500-1500 ps, LSC: 100-800 ps.)

Figure 2: Quantitative analysis of retinal autofluorescence lifetimes in retinal degeneration models. $\mathrm{MNU}(r e d)$ and $\mathrm{NalO}_{3}$ (blue)) as well as healthy control mice (ctrl, green) are depicted over the course of the experiment (day 3, 7, 14 and 28). Fluorescence lifetimes were measured in a short $(A)$ and a long $(B)$ spectral channel $(n=10$ for MNU and $\mathrm{NalO}_{3}, \mathrm{n}=6$ for the control group). Data is pooled from two independent experiments; Boxplots: box $=$ median $\pm 25 \%$, whiskers $=10 / 90 \%$ ).

Figure 3: Analysis of the two lifetime components $T 1$ and $T 2$ (long spectral channel). For illustration of the interaction of the short lifetime component $T 1$ and the long component T2, a distribution histogram is shown for each experimental group (control, $\mathrm{MNU}$ and $\mathrm{NaIO}_{3}$ ) at the last time point (d28). The distribution histogram shows specific lifetime clouds for each experimental condition.

Figure 4: Comparison of optical coherence tomography (OCT) imaging and histological sections (H\&E staining). Representative examples of the murine retina at experimental day 28 of control $(A)$ and pharmacologically induced retinal degeneration with $\mathrm{MNU}(\mathrm{B})$ or $\mathrm{NaIO}_{3}(\mathrm{C})$ are shown. For abbreviations see Table 1.

Figure 5: Total retinal thickness after injection of $\mathrm{MNU}$ respectively $\mathrm{NaIO}_{3}$ over time. Quantitative analysis of total retinal thickness values by optical coherence tomography (OCT) for each group at examination day 3, 7, 14, and 28 ( $\mathrm{n}=10$ for $\mathrm{MNU}$ and $\mathrm{NaIO}_{3}, \mathrm{n}=6$ for the control group (ctrl); Data is pooled from two independent experiments; Boxplots: box $=$ median $\pm 25 \%$, whiskers $=10 / 90 \%$ ).

Figure 6: Optical coherence tomography (OCT) analysis of the total retina and the photoreceptor layer (PR) over the course of experiment. A) Quantitative analysis of total retinal thickness values and PR thickness at day 3, 7, 14 and $28(n=4$ for each group). B) Retinal structure analysis using OCT: healthy retina of a control mouse 
(Abbreviations see Table 1). C) Degeneration of the murine retina over time in the $\mathrm{NalO}_{3}$ and the MNU model. Blue arrows mark remaining retinal pigment epithelium patches in the $\mathrm{NaIO}_{3}$ model. Red arrows indicate intact RPE and red asterisks indicate retinal detachment at location of the PR layer loss in the MNU model. Note that at day three in the $\mathrm{NaIO}_{3}$ degeneration model no major changes in the retinal architecture is detectable.

Figure 7: Nile Blue A staining for lipofuscin. Retina cross sections of control, MNU and $\mathrm{NaIO}_{3}$ treated mice stained with saturated Nile Blue A solution. An accumulation of lipofuscin (blue) was observed in the $\mathrm{NaIO}_{3}$ treated sample only. The scale bar equals 50 $\mu \mathrm{m}$. 


\section{References}

Baich, A. Ziegler, M., 1992. The effect of sodium iodate and melanin on the formation of glyoxylate. Pigment Cell Res. 5, 394-395.

Becker, W., 2012 Fluorescence lifetime imaging--techniques and applications. J. Microsc. 247, 119-136.

Delori, F., Greenberg, J. P., Woods, R. L., Fischer, J., Duncker, T., Sparrow, J., Smith, R. T., 2011. Quantitative measurements of autofluorescence with the scanning laser ophthalmoscope. Invest. Ophthalmol. Vis. Sci. 52, 9379-9390.

Delori, F. C., Dorey, C. K., Staurenghi, G., Arend, O., Goger, D. G., Weiter, J. J., 1995. In vivo fluorescence of the ocular fundus exhibits retinal pigment epithelium lipofuscin characteristics. Invest. Ophthalmol. Vis. Sci. 36, 718-729.

Dysli, C., Dysli, M. , Enzmann, V., Wolf, S., Zinkernagel, M. S., 2014. Fluorescence lifetime imaging of the ocular fundus in mice. Invest. Ophthalmol. Vis. Sci. 55, 72067215.

Dysli, C., Enzmann, V., Sznitman, R., Zinkernagel, M. S., 2015. Quantitative analysis of mouse retinal layers using automated segmentation of spectral domain optical coherence tomography images. Transl. Vis. Sci. Technol. 4, Article 9.

Dysli, C., Quellec, G., Abegg, M., Menke, M. N., Wolf-Schnurrbusch, U., Kowal, J., Blatz, J., La Schiazza, O., Leichtle, A. B., Wolf, S., Zinkernagel, M. S., 2014. Quantitative analysis of fluorescence lifetime measurements of the macula using the fluorescence lifetime imaging ophthalmoscope in healthy subjects. Invest. Ophthalmol. Vis. Sci. 55, 2106-2113.

Dysli, C., Wolf, S., Hatz , K., Zinkernagel, M. S., 2016. Fluorescence Lifetime Imaging in Stargardt Disease: Potential Marker for Disease Progression. Invest. Ophthalmol. Vis. Sci. 57, 832-841.

Dysli, C., Wolf, S., Zinkernagel, M. S., 2015. Fluorescence lifetime imaging in retinal artery occlusion. Invest. Ophthalmol. Vis. Sci. 56, 3329-3336.

Dysli, C., Wolf, S., Zinkernagel, M. S., 2016. Autofluorescence Lifetimes in Geographic Atrophy in Patients With Age-Related Macular Degeneration. Invest. Ophthalmol. Vis. Sci. 57, 2479-2487.

Ebneter, A., Agca, C., Dysli, C., Zinkernagel, M. S., 2015. Investigation of retinal morphology alterations using spectral domain optical coherence tomography in a mouse model of retinal branch and central retinal vein occlusion. PLoS One. 10, e0119046. 
Enzmann, V., Row, B. W., Yamauchi, Y., Kheirandish, L., Gozal, D., Kaplan, H. J., McCall, M. A., 2006. Behavioral and anatomical abnormalities in a sodium iodate-induced model of retinal pigment epithelium degeneration. Exp. Eye Res. 82, 441-448.

Franco, L. M., Zulliger, R., Wolf-Schnurrbusch, U. E., Katagiri, Y., Kaplan, H. J., Wolf, S., Enzmann, V., 2009. Decreased visual function after patchy loss of retinal pigment epithelium induced by low-dose sodium iodate. Invest. Ophthalmol. Vis. Sci. 50, 4004-4010.

Hueck, W., 1912. Pigmentstudien. Beitr. Path. Anat. 54, 68-232.

Islam, M. S., Honma, M., Nakabayashi, T., Kinjo , M., Ohta, N., 2013. pH Dependence of the Fluorescence Lifetime of FAD in Solution and in Cells. Int. J. Mol. Sci. 14, 19521963.

Kaneko, H., Nishiguchi, K. M., Nakamura, M., Kachi, S., Terasaki, H., 2008. Characteristics of bone marrow-derived microglia in the normal and injured retina. Invest. Ophthalmol. Vis. Sci. 49, 4162-4168.

Kiuchi, K., Yoshizawa, K., Shikata, N., Moriguchi, K., Tsubura, A., 2002. Morphologic characteristics of retinal degeneration induced by sodium iodate in mice. Curr. Eye Res. 25, 373-379.

Korte, G. E., Rappa, E., Andracchi, S., 1991. Localization of alkaline phosphatase on basolateral plasma membrane of normal and regenerating retinal pigment epithelium. A cytochemical study in rabbits. Invest. Ophthalmol. Vis. Sci. 32, 31873197.

Lille, R. D., 1956. Nile blue staining technique for the differentiation of melanin and lipofuscins. Stain Tech. 31, 151-154.

Loguinova, M. Y., Zagidullin, V. E., Feldman, T. B., Rostovtseva, Y. V., Paschenko, V. Z., Rubin, A. B., Ostrovsky, M. A., 2009. Spectral characteristics of fluorophores formed via interaction between all-trans-retinal with rhodopsin and lipids in photoreceptor membrane of retina rod outer segments. Biochemistry, Moscow. Supplement Series A: Membrane and Cell Biology, 3, 134-143.

Lozano, D. C., Twa, M. D., 2013. Development of a rat schematic eye from in vivo biometry and the correction of lateral magnification in SD-OCT imaging. Invest. Ophthalmol. Vis. Sci. 54, 6446-6455.

Machalinska, A., Lejkowska, R., Duchnik, M., Kawa, M., Roginska, D., Wiszniewska, B., Machalinski, B., 2014. Dose-dependent retinal changes following sodium iodate administration: application of spectral-domain optical coherence tomography for 
monitoring of retinal injury and endogenous regeneration. Curr. Eye Res. 39, 10331041.

Reisenhofer, M., Balmer, J., Zulliger, R., Enzmann, V., 2015. Multiple programmed cell death pathways are involved in $\mathrm{N}$-methyl-N-nitrosourea-induced photoreceptor degeneration. Graefes Arch. Clin. Exp. Ophthalmol. 253, 721-731.

Remtulla, S., Hallett, P. E., 1985. A schematic eye for the mouse, and comparison with the rat. Vision Res. 25, 21-31.

Sauer, L., Schweitzer, D., Ramm, L., Augsten, R., Hammer, M., Peters, S., 2015. Impact of Macular Pigment on Fundus Autofluorescence Lifetimes. Invest. Ophthalmol. Vis. Sci. 56, 4668-4679.

Schweitzer, D., 2010. Metabolic Mapping, in: Medical Retina, Holz, F. G., Spaide, R.F. (Eds.), Essentials in Ophthalmology. Springer, Berlin, Heidelberg, pp. 107-123.

Schweitzer, D., Gaillard, E. R., Dillon, J., Mullins, R. F., Russell, S., Hoffmann, B., Peters, S., Hammer, M., Biskup, C., 2012. Time-resolved autofluorescence imaging of human donor retina tissue from donors with significant extramacular drusen. Invest. Ophthalmol. Vis. Sci. 53, 3376-3386.

Schweitzer, D., Schenke, S., Hammer, M., Schweitzer, F., Jentsch, S., Birckner, E., Becker, W., Bergmann, A., 2007. Towards metabolic mapping of the human retina. Microsc. Res. Tech. 70, 410-419.

Sen, H. A., Berkowitz, B. A., Ando, N., de Juan, Jr., E., 1992. In vivo imaging of breakdown of the inner and outer blood-retinal barriers. Invest. Ophthalmol. Vis. Sci. 33, 3507-3512.

Skala, M. C., Riching, K. M., Gendron-Fitzpatrick, A., Eickhoff, J., Eliceiri, K. W., White, J. G., Ramanujam, N., 2007. In vivo multiphoton microscopy of NADH and FAD redox states, fluorescence lifetimes, and cellular morphology in precancerous epithelia. Proc. Natl. Acad. Sci. USA. 104, 19494-19499.

Sparrow, J. R., Gregory-Roberts, E., Yamamoto, K., Blonska, A., Ghosh, S. K., Ueda , K., Zhou, J., 2012. The bisretinoids of retinal pigment epithelium. Prog. Retin. Eye Res. 31, 121-135.

Tsubura, A., Yoshizawa, K., Kuwata, M., Uehara, N., 2010. Animal models for retinitis pigmentosa induced by MNU; disease progression, mechanisms and therapeutic trials. Histol. Histopathol. 25, 933-944.

Wan, J., Zheng, H., Chen, Z. L., Xiao, H. L., Shen, Z. J., Zhou., G. M., 2008. Preferential regeneration of photoreceptor from Muller glia after retinal degeneration in adult rat. Vision Res. 48, 223-234. 
Wang, J., lacovelli, J., Spencer, C., Saint-Geniez, M., 2014. Direct effect of sodium iodate on neurosensory retina. Invest. Ophthalmol. Vis. Sci. 55, 1941-1953.

Yang, Y., Ng, T. K., Ye, C., Yip, Y. W., Law, K., Chan, S. O., Pang, C. P., 2014. Assessing sodium iodate-induced outer retinal changes in rats using confocal scanning laser ophthalmoscopy and optical coherence tomography. Invest. Ophthalmol. Vis. Sci. $55,1696-1705$.

Yoon, Y. H., Marmor, M. F., 1993. Retinal pigment epithelium adhesion to Bruch's membrane is weakened by hemicholinium-3 and sodium iodate. Ophthalmic Res. 25, 386-392.

Yoshizawa, K., Nambu, H., Yang, J., Oishi, Y., Senzaki, H., Shikata, N., Miki, H., Tsubura, A., 1999. Mechanisms of photoreceptor cell apoptosis induced by N-methyl-Nnitrosourea in Sprague-Dawley rats. Lab. Invest. 79, 1359-1367. 
Figure 1

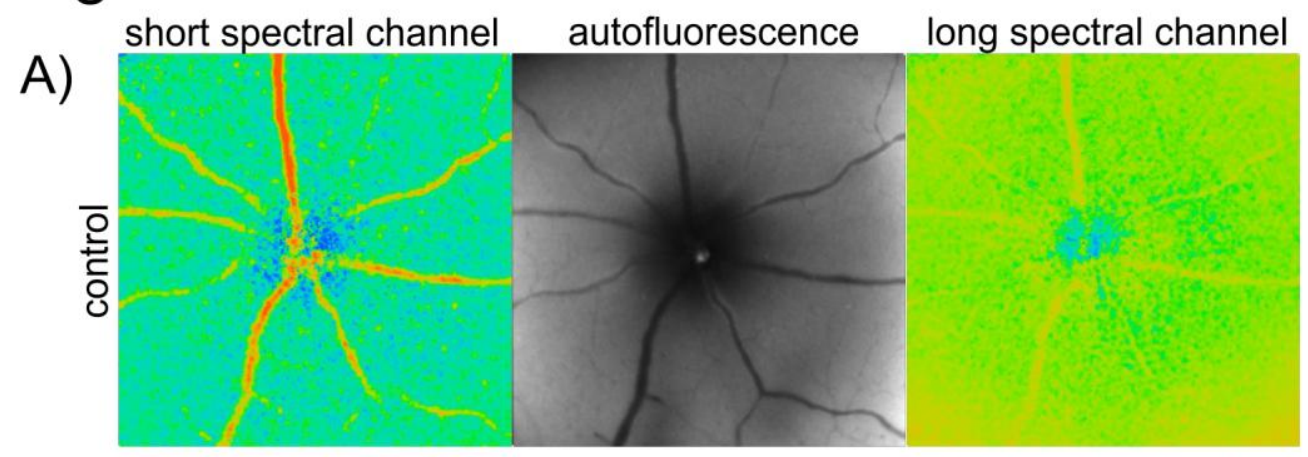

B)

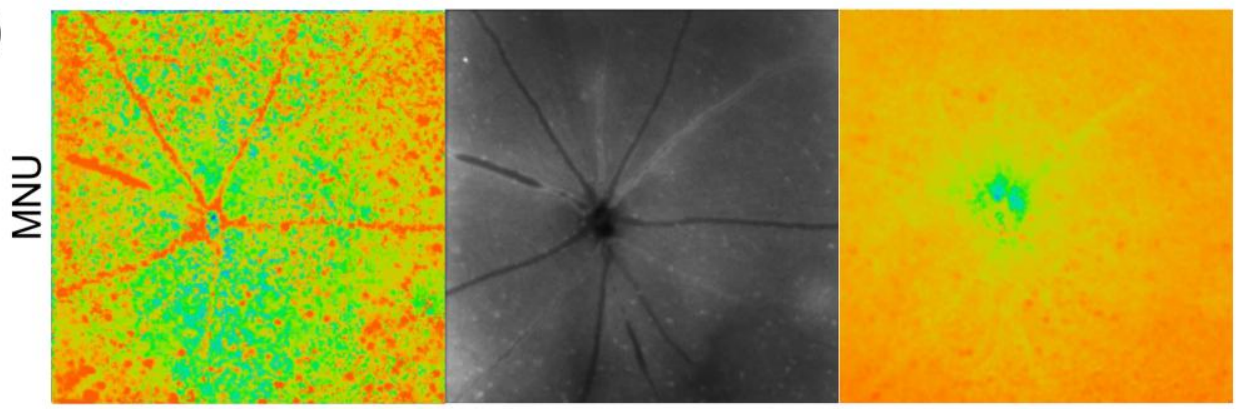

C)

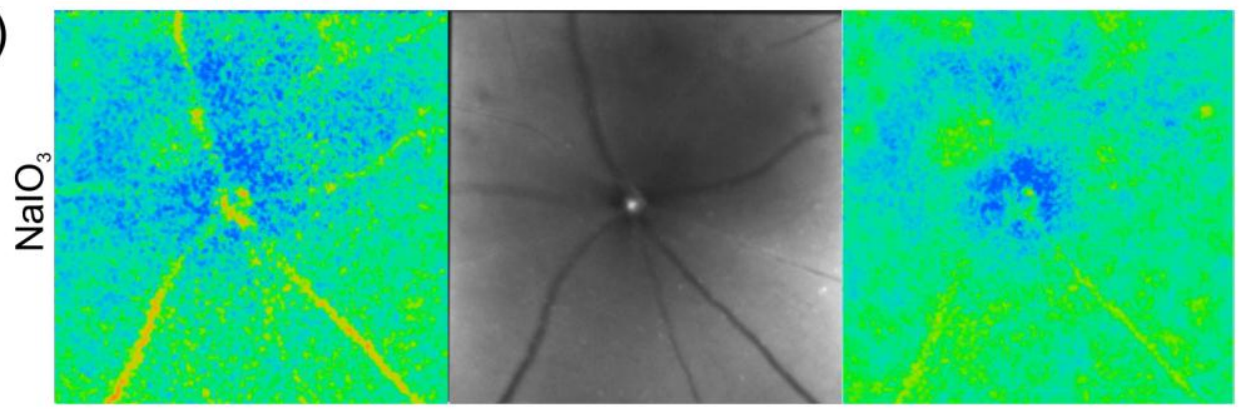

D)
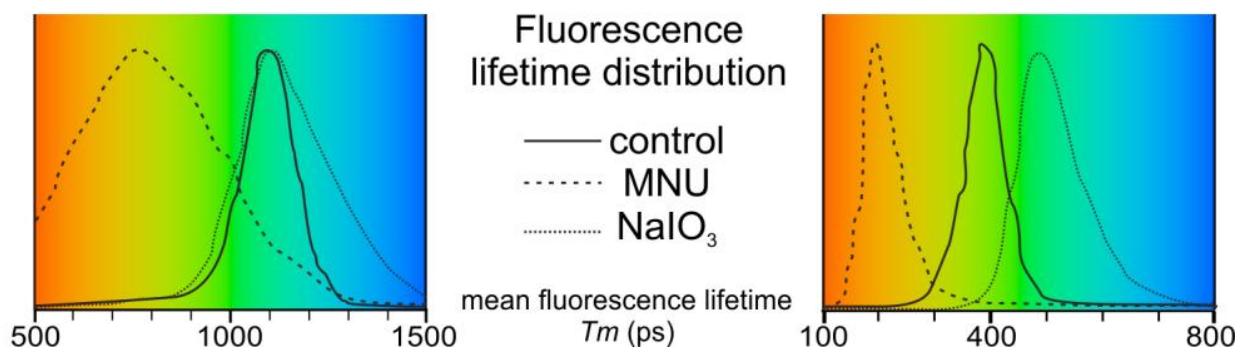
Figure 2
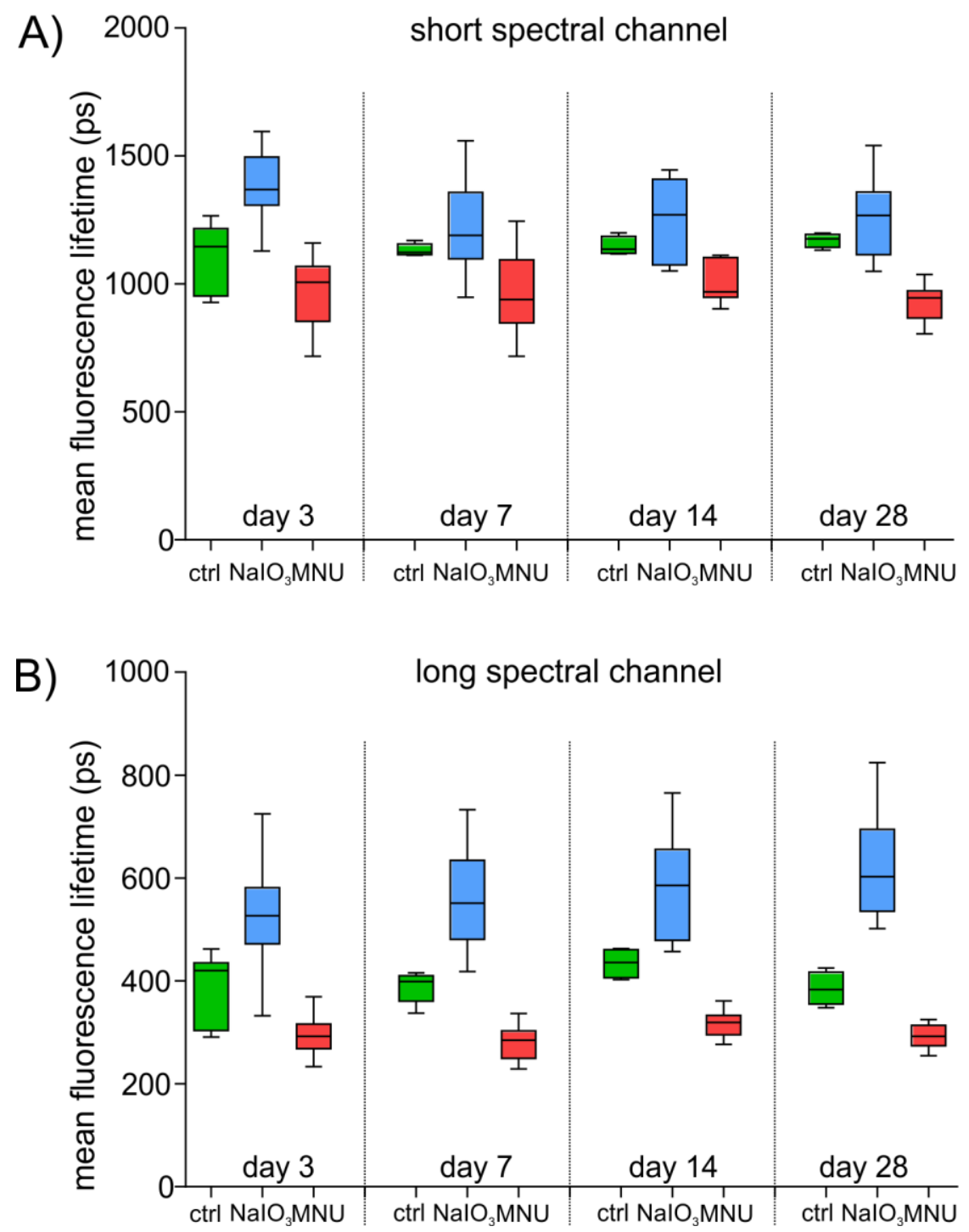
Figure 3

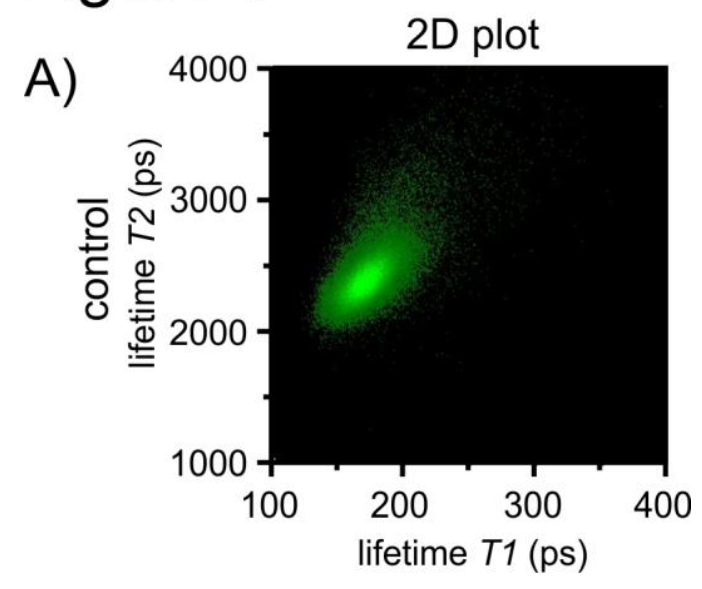

B)
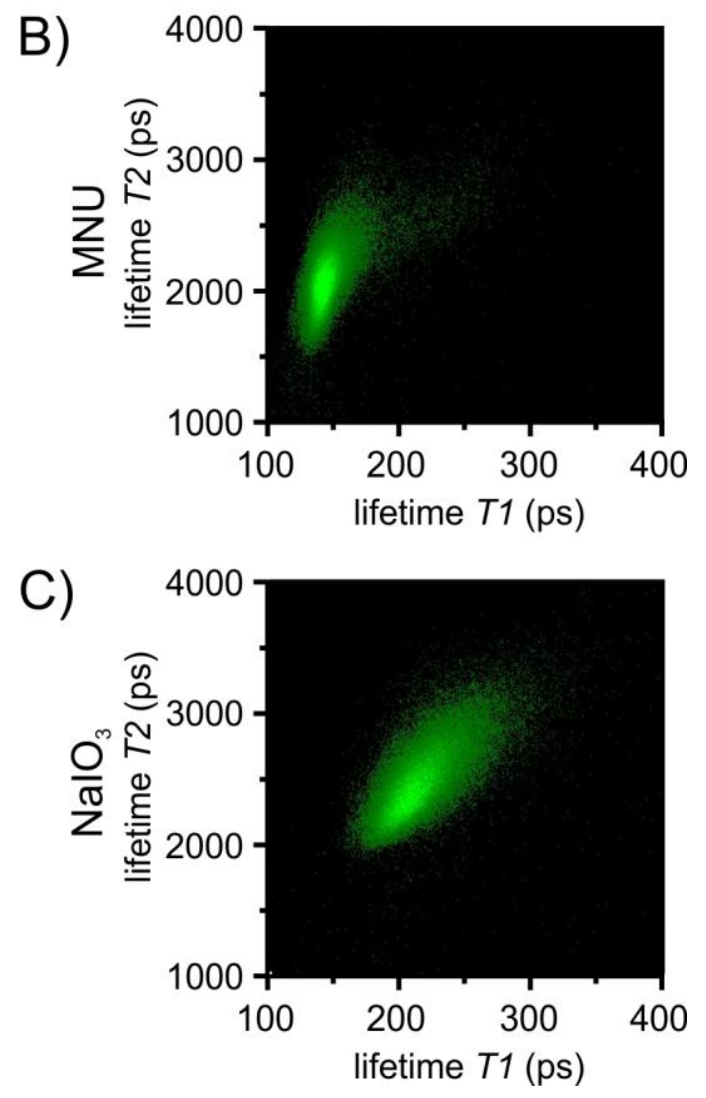
Figure 4

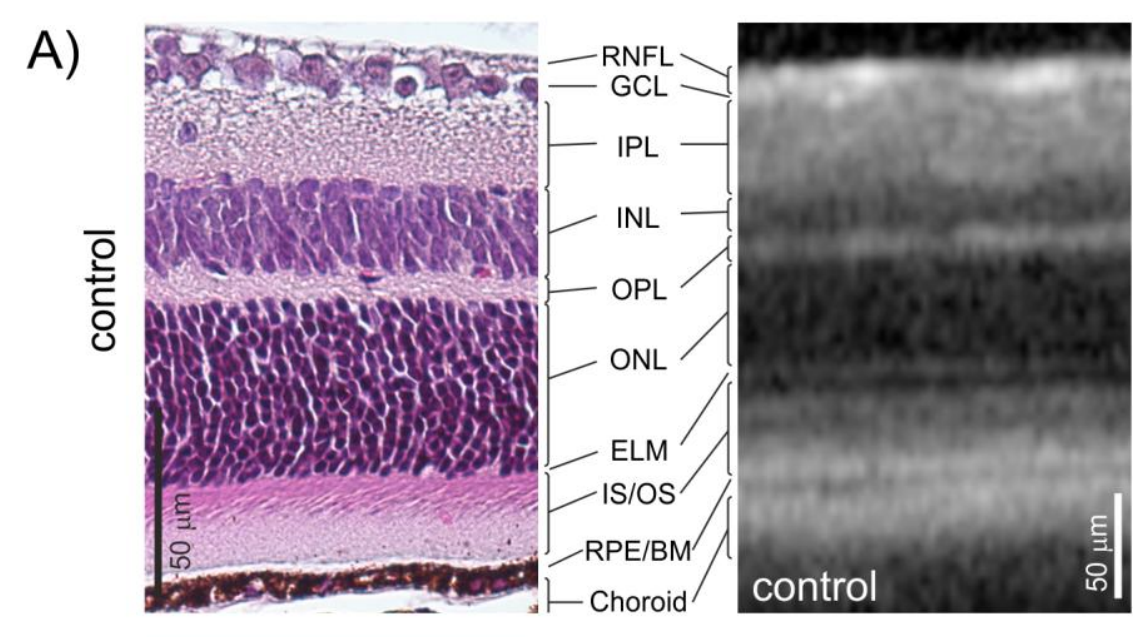

B)
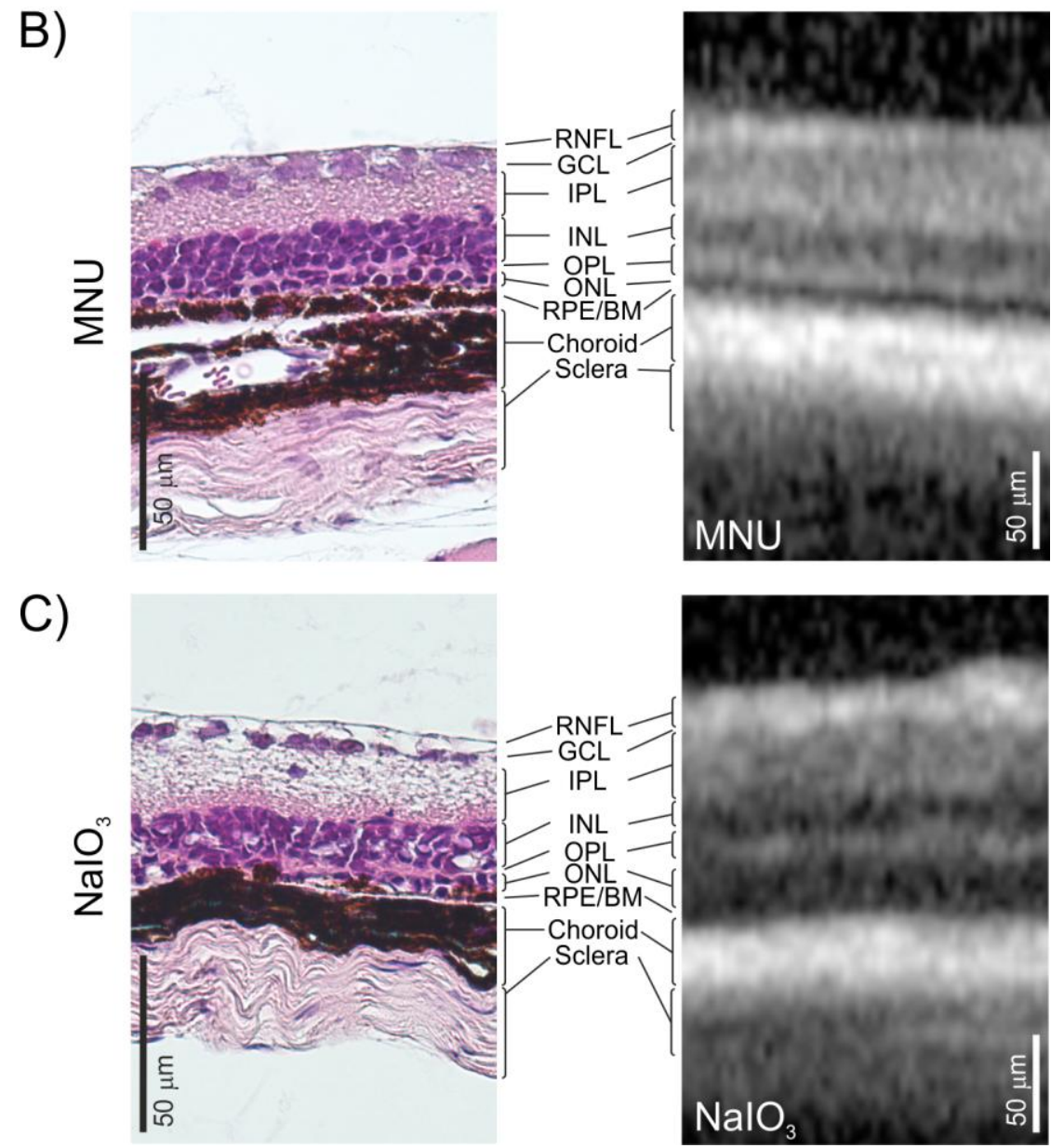
Figure 5

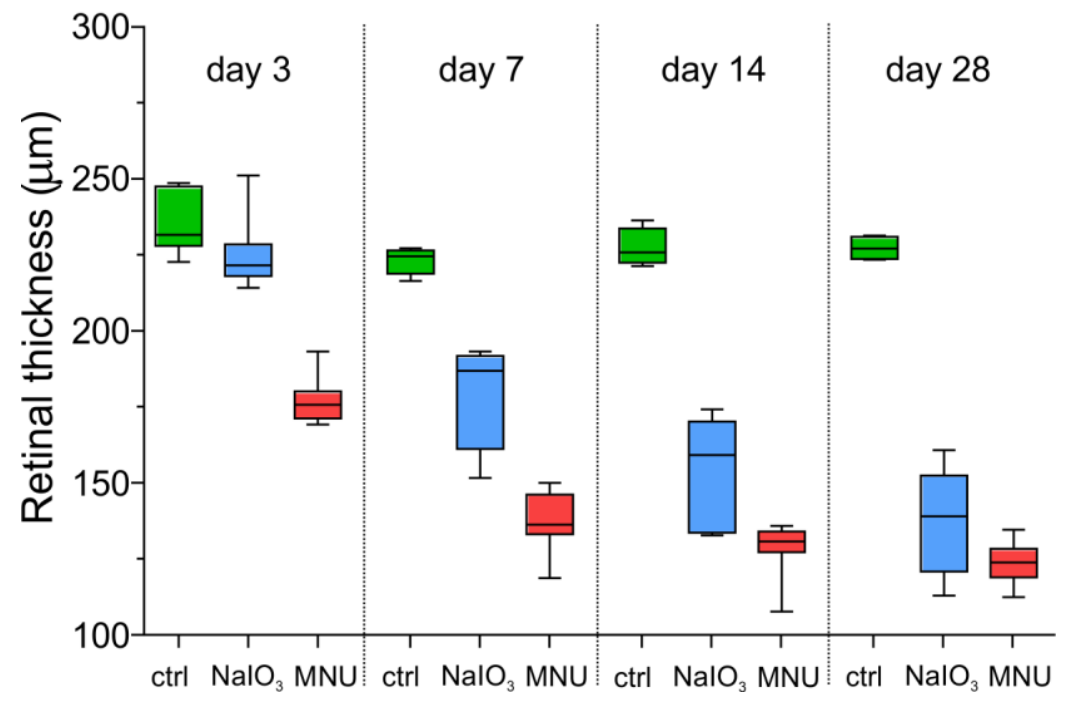


Figure 6

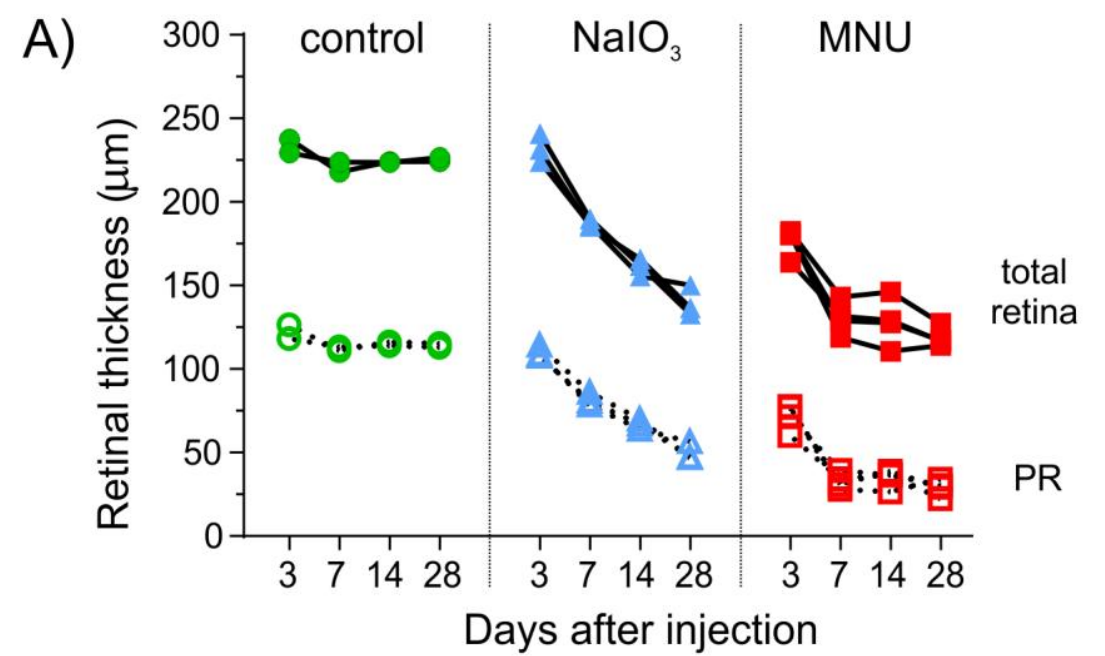

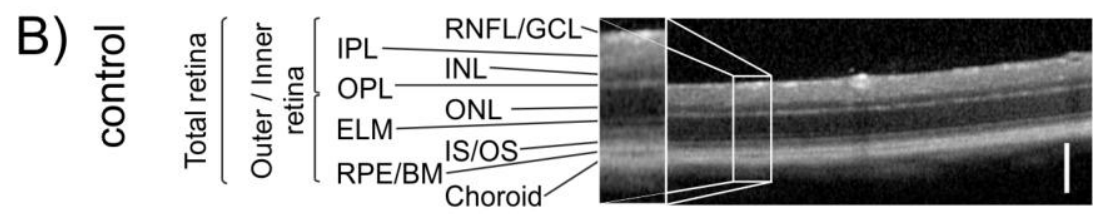

C)

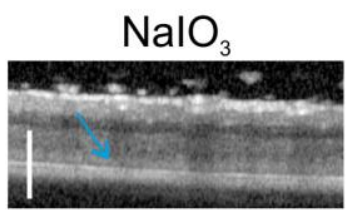

day

MNU

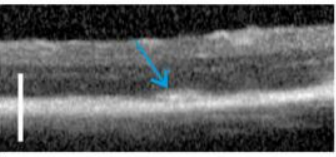

3
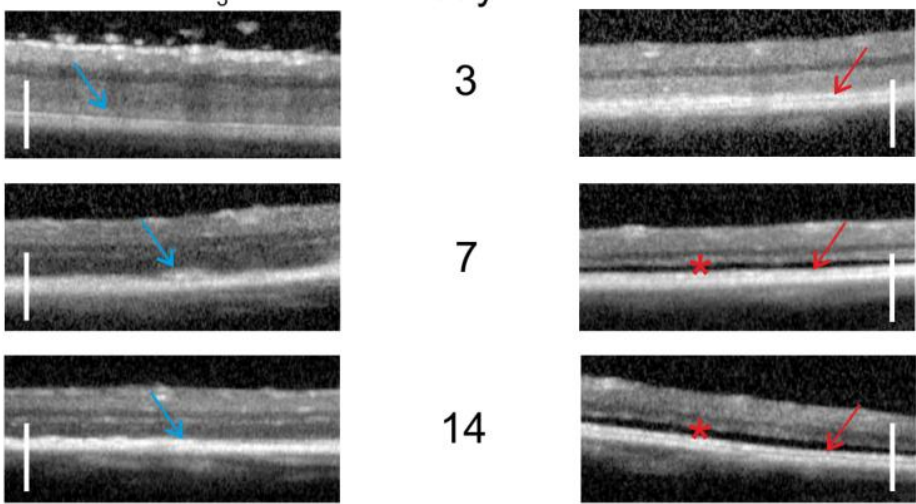

7

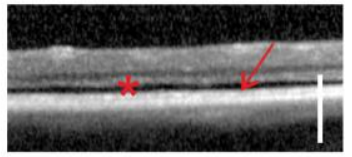

14
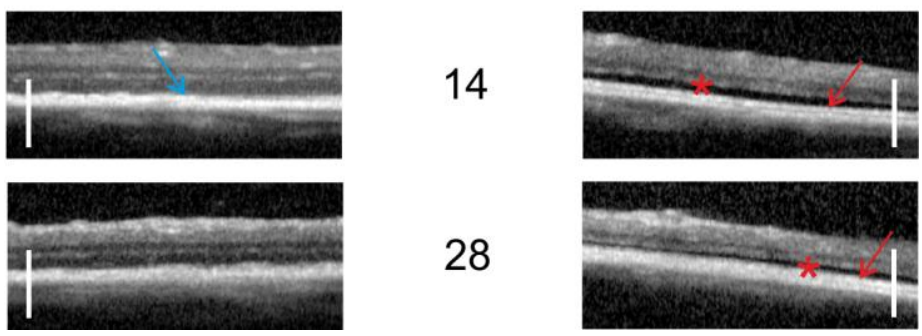

28

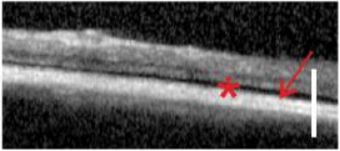



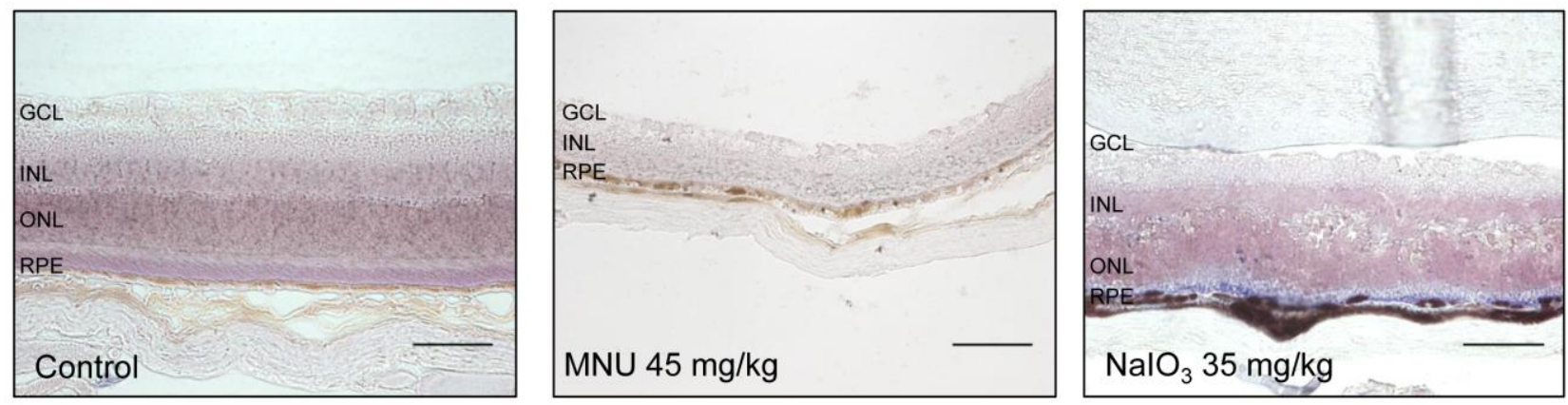